\title{
The short-term prognosis of severe primary infantile malnutrition
}

\author{
BY J. S. GARROW* \\ Medical Research Council, Tropical Metabolism Research Unit, \\ Famaica, West Indies \\ AND M. C. PIKE† \\ Medical Research Council Statistical Research Unit, University College \\ Hospital Medical School, London
}

(Received 22 Fune r966-Accepted 26 September 1966)

\begin{abstract}
I. Among a series of 343 children with severe primary malnutrition there were 248 for whom, on admission, there were reliable records of age, weight, height, liver size, severity of oedema, skin lesions and angular stomatitis, and concentration of total serum protein, haemoglobin and sodium. For eighty-four of these children the serum bilirubin concentration was also known.

2. The correlations of these characteristics of the children on admission, with mortality, and with the rate of recovery were investigated.

3. Age, weight, oedema and haemoglobin concentration were not significantly related to mortality or to rate of recovery. A multiple regression analysis showed that an increased serum bilirubin concentration and a decreased serum sodium concentration indicated a bad prognosis, and these two factors contributed almost the whole of the multiple correlation coefficient of 0.63 with respect to mortality and 0.59 with respect to speed of recovery.

4. We conclude that in our series of children death was more closely associated with liver failure or overhydration than with protein depletion, and suggest that the administration of too much protein or water to an acutely ill malnourished child may precipitate death.

5. 'This analysis shows that the mortality observed in our series cannot be adequately explained by any combination of the characteristics considered above; there must, therefore, be other factors of importance for which we do not have suitable measurements.
\end{abstract}

The mortality in severe infantile malnutrition is high and the cause of death is not well understood. In Jamaica a typical malnourished child is about $\mathrm{x}$ year old and weighs about $5 \mathrm{~kg}$; his liver may be of normal size or it may be grossly enlarged as a result of intense fatty infiltration, and he may or may not have oedema. Nutritional dermatoses occur, but they are not usually severe. Blood analysis may show a moderate to marked decrease in the haemoglobin, protein, sodium and potassium concentrations, and an increase in bilirubin and certain enzymes. All the above factors can be measured and statistically analysed, but other factors which seem clinically to be important in prognosis, such as the mental state of the child and the severity of infection, are difficult to record objectively.

Mexican workers (Gómez, Ramos-Galván, Frenk, Cravioto-Muñoz, Chávez \& Vázquez, 1956; Ramos-Galván \& Calderon, 1965$)$ have reviewed a series of 2401 children with 'third degree malnutrition' and have concluded that the age of the child, the

* Present address: MRC Laboratory, Mint Stables, St Mary's Hospital, London, W 2.

$\uparrow$ Present address: Department of Pathology, Makerere College, P.O. Box 2072, Kampala, Uganda. 
severity of malnutrition, and the presence of infection and electrolyte imbalance are the important factors in determining mortality. A malnourished child with a severe infection and profuse diarrhoea is very likely to die, whereas a more severely malnourished child without infection will probably survive. In any series which includes children with severe infections, therefore, the infection is likely to overshadow the effects of malnutrition itself.

This paper presents an analysis of the factors which seemed to determine the prognosis of a series of severely malnourished children who did not have severe infections, metabolic defects or other organic causes of failure to thrive. Between October 1956 and January 1962 about 500 malnourished children were admitted to the metabolic ward of the MRC Tropical Metabolism Research Unit in Jamaica. Out of this number 343 children fulfilled the criteria of severe primary malnutrition. These were, first, that the body-weight of the child (after allowing for oedema) was less than $75 \%$ of that of an average North American child of the same age (Nelson, 1959), and second, that from clinical assessment and response to treatment this weight deficit was primarily attributable to undernutrition.

The treatment of these children was along lines described elsewhere (Garrow, Picou \& Waterlow, r962); essentially the objectives are to treat infections with appropriate antibiotics, to correct electrolyte disturbances with intravenous fluids if necessary, and to give a diet supplying increasing amounts of nutrients until a level of $150 \mathrm{kcal}$ and $3.5 \mathrm{~g}$ protein per $\mathrm{kg}$ body-weight per day is reached. Up to about June 1960 the fluid intake of the children was usually $150 \mathrm{ml} / \mathrm{kg}$ day or more; thereafter following the demonstration by Smith (I960a) that even non-oedematous children had greatly increased body water, the fluid intake of the children was reduced to about $85 \mathrm{ml} / \mathrm{kg}$ day, and from that time I $\mathrm{mg}$ folic acid daily was given routinely to all children. The level of potassium supplementation used was inadequate by present standards; during the period covered by the series described here the normal supplement was 2.5 m-equiv. potassium per $\mathrm{kg}$ per day, whereas we would now give 6-8 mequiv. per $\mathrm{kg}$ per day to children with oedema (Garrow, 1965).

\section{METHODS}

In order to avoid bias, each child in the series was first assigned to one of four groups according to his clinical progress, the criteria being as follows.

Group I : died.

Group 2: recovered slowly; those children who failed to show definite improvement in clinical condition within the first 3 weeks in hospital.

Group 3: intermediate recovery; those children with a response between those of group 2 and group 4 .

Group 4: rapid recovery; those children who showed definite clinical improvement during the Ist week in hospital.

When this had been done the following information was abstracted from the case records on to punched cards:

(I) The age, weight and height of the child, the duration of illness, the period of 
breast feeding, the nature of the weaning diet, the size of the family and the position of the child in it, the age of the mother and whether the home was in an urban or a rural area.

(2) The clinical features observed on admission to hospital were graded into three degrees of severity. Oedema was classified as grade $\circ$ if no definite pitting could be demonstrated after pressure on the dorsum of the foot, as grade I if there was pitting of the legs but not of the arms, and as grade 2 if all limbs and trunk were involved. Liver enlargement was assessed by palpation in the supine position, and graded as grade $O$ if the edge was within I cm of the costal margin, as grade I if it was $2-5 \mathrm{~cm}$ below the ribs, and as grade 2 if it was $6 \mathrm{~cm}$ or more below them. Skin lesions and angular stomatitis were graded by inspection as grade 0,1 or 2 . Although the mental state of the child is a very valuable prognostic index, no satisfactory method was found of grading the fluctuating states of apathy, misery and irritability which are characteristic of the malnourished child, so this factor could not be included in the analysis.

(3) Measurements which were made on blood taken on admission to hospital included the haemoglobin and serum sodium, potassium, bicarbonate, total protein, bilirubin and alkaline phosphatase concentrations. Sometimes, as a result of laboratory error or difficulty in obtaining a blood sample, some of these measurements were not made, and this raised the possibility that missing values might introduce bias into the results. However, the mortality and clinical features of children in whom values were missed were not significantly different from those of the remainder of the series (see Table r), so that it is unlikely that this factor caused serious bias.

In order to determine which factors were likely to be significantly related to clinical progress, the series was first analysed by each of the punched card entries in turn. Each factor was divided into high, middle and low ranges with boundaries set so that approximately one-third of the series lay in each group. The clinical progress of the high and low groups was then compared, and if there was clearly no significant difference this factor was not included in the subsequent analysis. As a result of this rough screening procedure, it was decided to continue with a multiple regression analysis of the following observations: (I) age on admission, (2) minimum weight (i.e. the lowest weight recorded for the child during the complete course of treatment, discussed on p. 162) as a percentage of reference weight for age, (3) minimum weight as percentage of reference weight for height, (4) liver size on admission, (5) oedema on admission, (6) skin lesions on admission, (7) lesions of buccal mucosa on admission, (8) haemoglobin concentration on admission, (9) serum sodium concentration on admission, (Io) serum total protein concentration on admission, (II) serum bilirubin concentration on admission.

For 248 children there were complete records for the first ten factors (hereafter referred to as patients with complete records). These children were all less than 24 months old. There were in addition five children with complete records who were over this age, but they were excluded because they were so much older than the main body of children that their inclusion in regression analyses involving age might give misleading results. The clinical progress of those with complete and incomplete records is shown in Table I. There is clearly very little difference between the two groups. 
Measurements of the serum bilirubin concentration on admission to hospital were available for only eighty-four of these 248 children, so this factor was examined by separate regression analyses. The clinical progress of those with and without bilirubin measurement is also shown in Table I. The differences between the two groups
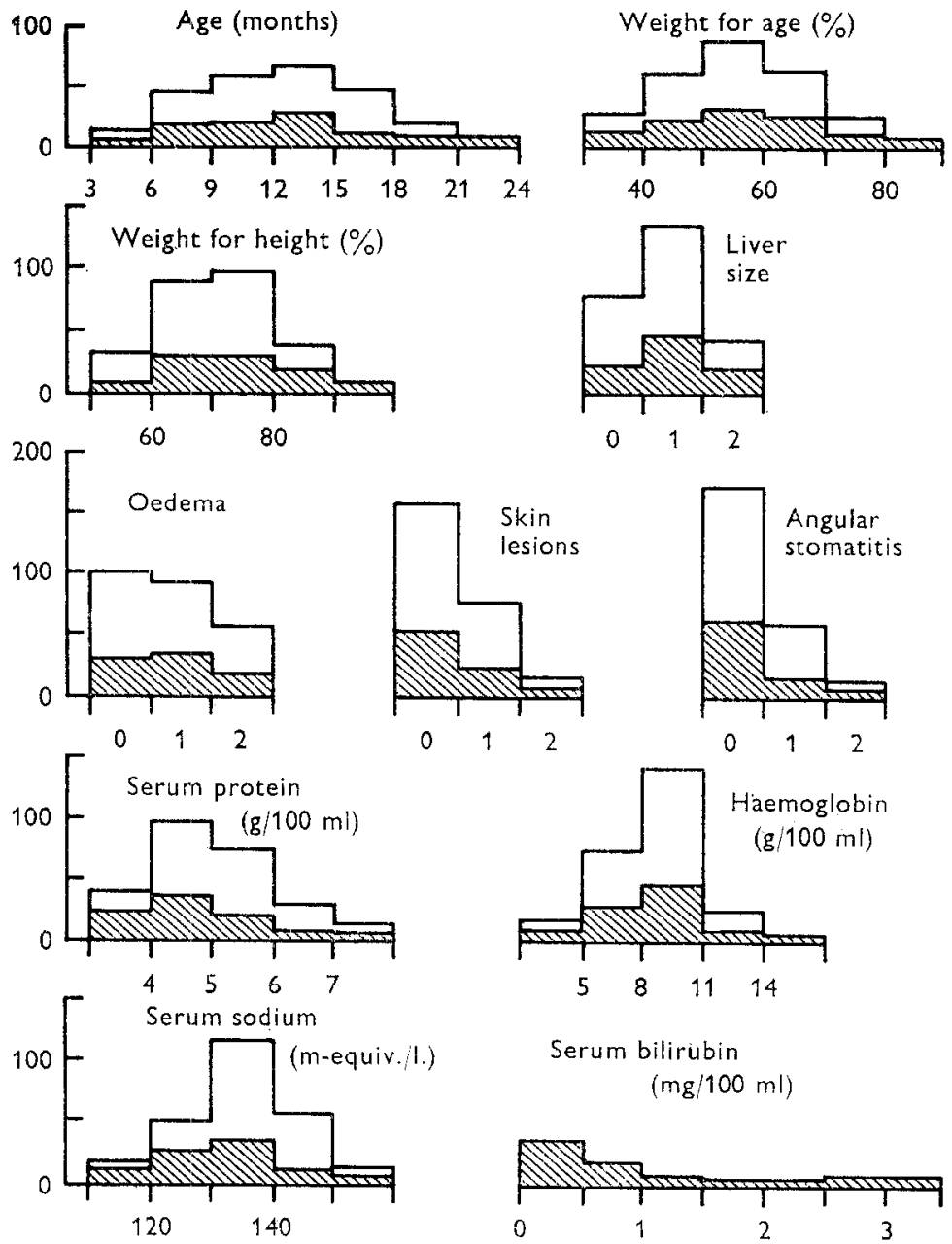

Fig. I. Histogram showing the distribution of characteristics among 248 malnourished children on admission to hospital. The shaded part of the histogram represents the distribution of these characteristics among the eighty-four children in whom the serum bilirubin concentration on admission was measured.

is not statistically significant, and although the patients with known bilirubin values were on average those admitted later in the period under study, there is no reason to suppose that the observed difference was not solely due to chance. Fig. I shows the distribution of presenting features of the 248 patients and of the subsample of eightyfour patients with known bilirubin values. 


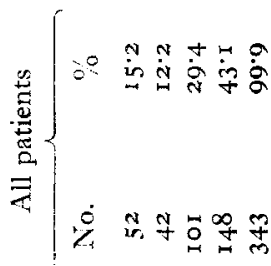
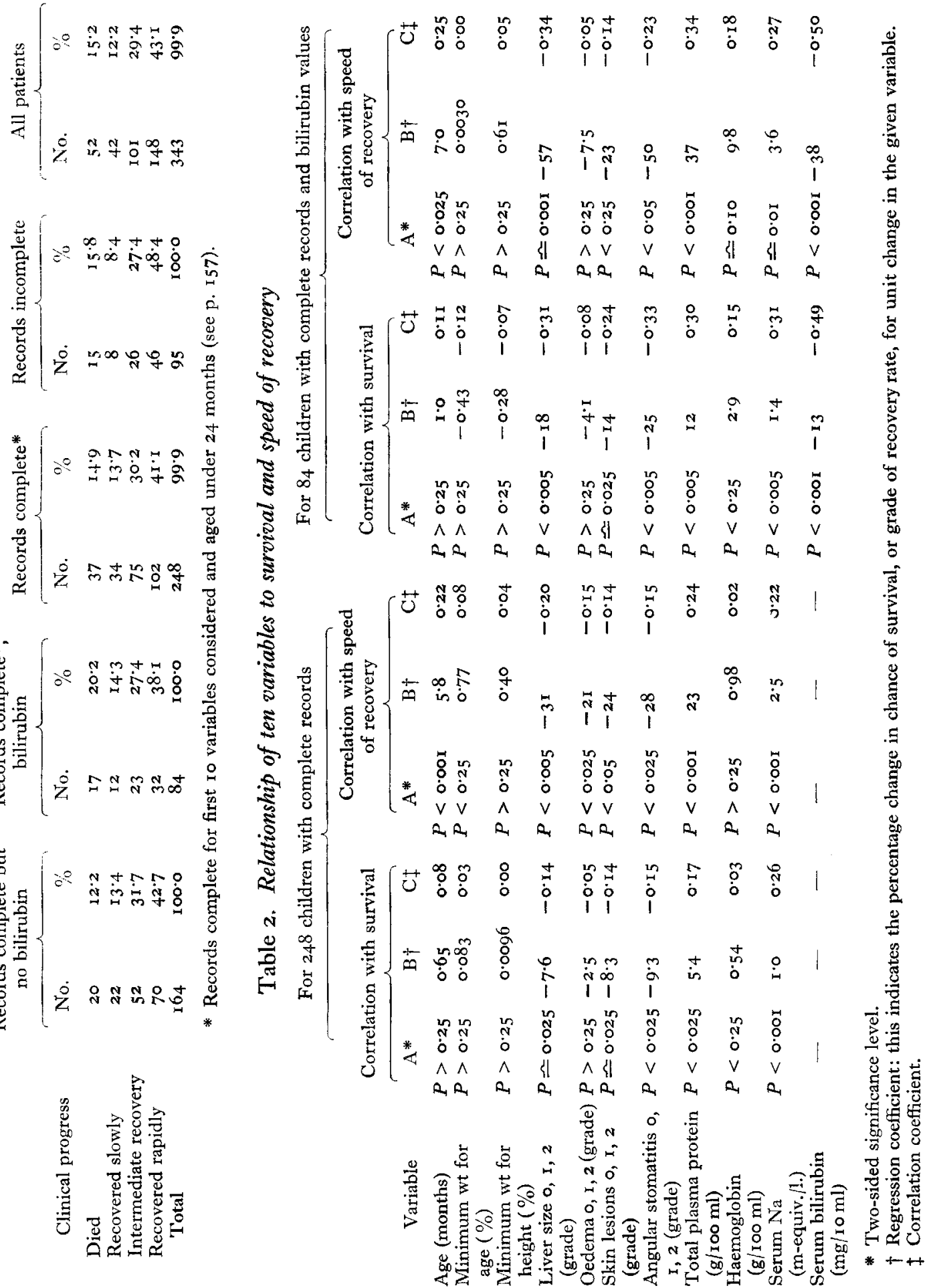


\section{RESULTS}

\section{Survival and progress}

The analysis of the prognosis of the patients was made in two ways. We considered first survival, i.e. whether or not the child died, and secondly progress, i.e. noting in addition the rate of recovery of the children who survived. For the analysis of survival we scored death $=0$ and recovery $=\mathrm{I}$, while for the progress analysis we scored death $=1$, slow recovery $=2$, intermediate recovery $=3$, rapid recovery $=4$. The scoring for the progress analysis is obviously somewhat artibrary, but a different choice of scores is unlikely to lead to markedly different conclusions.

Table 2 gives the results of simple (one factor at a time) regression analyses of survival and progress against the clinical features. The main points to be noted in these tables are as follows. (a) Concerning survival: age, weight, oedema and haemoglobin by two-sided test were not significantly $(P>0.05)$ correlated with survival either in the main series or in the subgroup with known bilirubin values. $(b)$ Concerning progress: only weight and haemoglobin were not significantly correlated with progress in the whole series of 248 children.

These analyses show that the factor most strongly correlated with survival (correlation coefficient -0.49 ) was bilirubin concentration. After this came, in decreasing order of significance, angular stomatitis, serum sodium concentration, liver size, total plasma protein concentration and skin lesions. However, after allowing for bilirubin, this order of decreasing significance is changed to serum sodium, angular stomatitis, skin lesions and total plasma protein (all still significant at the $5 \%$ level), and liver size is no longer significantly correlated with survival. On this basis, we did a series of multiple regression analyses, which first took in bilirubin and then serum sodium, and proceeded step by step, the best new variable being added in, until no further feature significantly $(P<0.05)$ improved the correlation. In this way it was found that as bilirubin, serum sodium, and angular stomatitis were allowed for, the multiple regression coefficient rose successively from 0.49 to 0.56 to 0.63 . Thereafter no further feature was significantly correlated with survival.

The picture is more dramatic when progress is analysed. After bilirubin had been allowed for, only total plasma protein and serum sodium were significantly $(P<0.05)$ correlated with progress, and when either of these two features was also allowed for no other feature was significantly correlated with progress. Taking into account bilirubin, serum sodium and total plasma protein gives a multiple correlation coefficient of 0.59 .

It has been stated above that, on simple regression analysis, factors such as liver size were significantly related to both survival and speed of recovery; however, liver size contributes nothing to the multiple regression. This situation arises because liver size is very significantly related to bilirubin level, and when bilirubin has been taken into account the finding of an enlarged liver contributes no further information concerning the prognosis. It is necessary, therefore, if results are to be interpreted correctly, to consider correlations among the variables themselves, as well as between single variables and clinical progress. The correlations between variables are shown in 





Table 3 ; the whole series has been used to calculated correlation coefficients among the first ten variables, and the right-hand column of the table shows the correlation between these variables and the bilirubin level in those eighty-four children in whom bilirubin was measured. In reading the table it is necessary to pay attention to the sign before the correlation coefficient: for example, the older the child was the higher was his plasma protein $(P<0.05)$ but the lower was his haemoglobin $(P<0.01)$. An increase in the minimum weight of the child (both for age and height) was highly significantly correlated $(P<\mathrm{O} \cdot \mathrm{OI})$ with an increase in liver size and severity of oedema; and liver enlargement, oedema and skin lesions were associated with a reduction of plasma protein level $(P<0.0 \mathrm{I})$ and an increase in angular stomatitis $(P<0.01)$.

\section{DISCUSSION}

One of the fundamental problems in the study of infantile malnutrition is that we have no good way of assessing which of a group of children is most severely malnourished. Of the available measurements, body-weight is probably the most reliable, so it is to be expected that the children most underweight are the most severely undernourished, and hence the most likely to die of malnutrition. In fact Gómez et al. (1956) and Kahn (I959a) have found an association between weight deficit and mortality, but in our series the correlation is not significant either with weight for age or weight for height even when the minimum weight is used for the calculation. Minimum weight differed from weight on admission only in children who were oedematous on admission. Oedema is weakly associated with poor clinical progress (see Table 2), so that if an association had not been found between weight on admission and clinical progress this might have arisen because some very underweight (and hence bad risk) children had their weight on admission augmented by oedema fluid. However, by using minimum weight the chance of falling into this error is decreased, and still the correlation between weight and prognosis was not significant. Hypoproteinaemia, which is another index of protein depletion (Schendel, Hansen \& Brock, I962), also makes a poor showing as a prognostic index, compared with hyperbilirubinaemia and hyponatraemia. The series of children described here all certainly had severe malnutrition, but it appears that factors other than protein depletion were responsible for the majority of the deaths.

The finding that the most important prognostic index was the bilirubin value is rather surprising. Ramos-Galván \& Calderon (1965) attach importance to age, electrolyte imbalance, the presence of infection and the degree of weight deficit. Our criteria excluded those children with severe infections. The age and weight of the child did not contribute significantly to the chance of survival in the series, although among survivors the older children recovered more rapidly. However, electrolyte imbalance was important: this is shown by the fact that the serum sodium was second only to bilirubin in correlation with survival; the regression coefficient of $\mathrm{I} \cdot 0$ shown in Table 2 means on average, each m-equiv./l., that the serum sodium increases or decreases implies an increase or decrease of $I \%$ in the chance that the child will survive. It is unfortunate that reliable figures for serum potassium were not available for this analysis, 
since a smaller series from the same Unit (Smith, I $960 b$ ) showed that children. who died had a significantly lower serum potassium concentration than survivors. However, very severe potassium depletion may occur in malnourished children with normal serum concentrations (Garrow, I965); serum potassium measurements may accordingly sometimes be very misleading.

Kahn (1959a) analysed prognostic criteria in a series of 100 children with severe protein malnutrition, but excluded any who did not show either oedema or nutritional dermatosis. He concluded that factors which indicated a bad prognosis were a bodyweight less than $50 \%$ of the Boston 5 oth percentile for age, acute dermatosis, dehydration with hyponatraemia and hypokalaemia, enlargement of the liver and hypothermia. He found that age was not important, nor was retarded growth in height, and this agrees with our findings. Also in agreement is the fact that liver enlargement and skin lesions are associated with a bad prognosis, but not nearly so strongly as hyponatraemia and raised serum bilirubin. Kahn used clinical criteria to assess 'dehydration' and it is difficult to make a valid comparison between his findings and those reported here concerning this condition.

Smith (1963) also noted that hyponatraemia was associated with a high mortality, and there is good reason to suppose that therapeutic overhydration is a potent cause of death in malnourished children. Much has been said about the importance of 'rehydrating' these children (Jelliffe, 1961; Kahn, 1959a, b), but it is known that a malnourished child, even when he has gastroenteritis and shows the sunken eyes and lax skin of 'dehydration', in fact contains a normal or increased percentage of body water (Smith, 1960a). The pediatrician has to be careful that he does not give such a child too much fluid and thus produce the 'electrolyte imbalance' which is so dangerous.

Gómez and his colleagues in Mexico have studied the importance of numerous factors in malnourished children. In a paper dealing with liver function tests (Gómez, Ramos-Galván, Bienvenú \& Cravioto-Muñoz, 1950) they give the results of cephalin cholesterol, thymol turbidity and bromsulphalein clearance, and conclude that none of these are altered by malnutrition, although some abnormal turbidity tests were found in children with infections. They report that the indirect van den Burgh test for bilirubin was negative in all children, but the direct test gave values of $0.07-1 \cdot 37 \mathrm{mg} /$ I00 $\mathrm{ml}$ in fifty-eight malnourished children. The value found in fifty control children was $0.22 \pm 0.02 \mathrm{mg} / 100 \mathrm{ml}$. They conclude that the malnourished children all had serum bilirubin concentrations within the normal range, and do not relate the biljrubin level to the clinical progress of the malnourished children.

McLean (1962, 1965) pointed out that, in Jamaican children, a combination of a raised serum bilirubin concentration and an increase in the serum concentration of enzymes of hepatic origin was associated with a very high mortality. This syndrome often occurs in children with enlarged fatty livers, and once it is established it is extremely difficult to treat successfully. On the other hand, children with fatty livers but normal bilirubin and enzyme levels usually recover slowly but steadily. The analysis reported here indicates that the importance of raised bilirubin levels is not confined to those children showing this syndrome of hepatic failure; even when the 
whole series is considered, including those children in whom there was no liver enlargement, the serum bilirubin stands out as the best prognostic index.

These findings have a direct bearing on the therapy of severely malnourished children. In the past, emphasis has been given to rehydration and the administration of protein (Sénécal, I958; Gómez, Ramos-Galván, Cravioto \& Frenk, r958). We now find that the malnourished children most likely to die are those with hyponatraemia and raised bilirubin levels, and that these signs indicate expansion of extracellular water and impairment of liver function respectively. A rational way to treat a child with overhydration and incipient liver failure is to restrict his intake of water and protein. The restriction of fluid intake has been advocated by Paque (1958), and moderate protein intakes are very effective in curing malnourished children (Waterlow \& Wills, I960; Dumm, Webb, Periera, Begum \& Isaac, I963) without causing the 'recovery syndrome' which seems to occur only in children who are excessively fed (Gómez, Ramos-Galván, Cravioto \& Frenk, 1955).

In the Mexican series of cases approximately half the deaths occurred within $4^{8} \mathrm{~h}$ of admission to hospital, and Trowell, Davies \& Dean (1954) exclude such deaths when they discuss mortality statistics; the implication is that those children died before treatment could take effect. It is, of course, true that some children arrive in hospital in a moribund condition and die before any treatment is given, but if a child dies after treatment has been started we must consider the possibility that this treatment precipitated death, rather than failed to avert it. In view of the findings described in this paper it is recommended that particular care should be taken not to aggravate liver failure or hyponatraemia by overenthusiastic administration of protein or water to the very ill malnourished child, especially during the first few days in hospital.

Finally, attention may be drawn to the fact that, if the various characteristics listed in Table 2 included all the factors of importance in assessing prognosis, it should be possible to obtain a multiple correlation coefficient between these factors and clinical progress which approaches $\mathrm{I} \cdot \mathrm{O}$; in fact the best multiple correlation coefficient attainable is one of 0.63 for survival, and of 0.59 for rate of recovery. So we are, perhaps, about half-way towards understanding what determines the fate of a malnourished child.

One of us (J.S.G.) wishes to thank his colleagues on the medical and nursing staff of the MRC Tropical Metabolism Research Unit who made the observations on which this report is based, and Dr K. Garrow, Mrs E. Standard, SRN, and Mr I. Moseley who helped to abstract these data for preliminary analysis.

\section{REFERENCES}

Dumm, M. E., Webb, J. K. G., Pereira, S., Begum, A. \& Isaac, T. (1963). Int. Congr. Nutr. vi. Edinburgh p. 87.

Garrow, J. S. (1965). Lancet ii, 455 .

Garrow, J. S., Picou, D. \& Waterlow, J. C. (1962). W. Indian med. F. 11, 217.

Gómez, F., Ramos-Galván, R., Bienvenú, B. \& Cravioto-Muñoz, J. (1950). Boln. Hosp. Inf. Mexico 7, 485 .

Gómez, F., Ramos-Galván, R., Cravioto, J. \& Frenk, S. (1955). Adv. Pediat. 7, 13 I.

Gómez, F., Ramos-Galván, R., Cravioto, J. \& Frenk, S. (r958). Ann. N. Y. Acad. Sci. 69, 969. 
Gomez, F., Ramos-Galván, R., Frenk, S., Cravioto-Munoz, J., Chávez, R. \& Vázquez, J. (1956). F. trop. Pediat. 2, 77.

Jelliffe, D. B. (196r). F. trop. Pediat. 7, 29.

Kahn, E. (1959a). Am. F. clin. Nutr. 7, 161.

Kahn, E. (1959b). S. Afr. med. F. 33, 501 .

McLean, A. E. M. (1962). Lancet ii, 1292.

McLean, A. E. M. (1965). Clin. Sci. 30, 129.

Nelson, W. E. (1959). Textbook of Pediatrics, 7th ed., p. 50. Philadelphia: W. B. Saunders.

Paque, C. (1958). Maroc. méd. 37, 1063.

Ramos-Galván, R. \& Calderon, J. M. (1965). Am. F. clin. Nutr. r6, 35 I.

Schendel, H. E., Hansen, J. D. L. \& Brock, J. F. (1962). S. Afr. F. Lab. clin. Med. 8, 23.

Sénécal, J. (1958). Ann. N.Y. Acad. Sci. 69, 916.

Smith, R. (1960a). Clin. Sci. 19, 275.

Smith, R. (1960b). Total body water in infantile malnutrition. MD Thesis, University of Cambridge Smith, R. (1963). Lancet i, 77I.

Trowell, H. C., Davies, J. N. P. \& Dean, R. F. A. (1954). Kwashiorkor. London: Edward Arnold.

Waterlow, J. C. \& Wills, V. G. (1960). Br. F. Nutr. 14, 183 . 